\title{
Improving the Video Data Lookup Efficiency in Peer-to-Peer Live Streaming Network
}

\author{
Zhuo Chen, Gang Feng, and Yi Lu
}

\begin{abstract}
DHTs have been recently used in Peer-to-Peer (P2P) live streaming systems to improve video playback continuity and data segment availability. However, existing solutions are not especially optimized for live streaming applications. There are still some challenging issues to be addressed, especially the low search efficiency for time-sensitive applications. In this paper, we propose an efficient one-hop DHT lookup framework called Streaming DHT (SDHT), dedicated to supporting media data compensation for real-time streaming applications in P2P networks. SDHT framework includes an One-hop DHT implementation solution Enhanced Event Detection and Reporting Algorithm (EDRA+) and a distributed Index Mapping and Management Mechanism (DIMM). We conduct intensive simulation experiments based on OverSim to validate the effectiveness of SDHT and evaluate its performance. Numerical results show that SDHT can significantly improve the searching efficiency and media data availability for live streaming applications, with a low overhead.
\end{abstract}

Index Terms-Internet, peer-to-peer (P2P), live streaming, one-hop DHT.

\section{INTRODUCTION}

Existing P2P streaming systems can be roughly classified into two categories: Tree-based and Mesh-based systems. Tree-based streaming systems usually organize nodes into a single tree or multi-tree structure, where the root of the tree is the media source and data segments are always pushed from parents down to the children. Most practical P2P streaming systems such as PPLive, PPStream, SopCast belong to mesh-based system, where each peer may have multiple neighbor peers. Peers periodically exchange Buffer Map (BM) messages with each other and peers can request stream data from its neighbors according to the received BM. No matter in the tree-based or mesh-based systems, a peer can only retrieve streaming data from limited peers which we call local neighbors. Because tree-based systems are fragile to network dynamics and data dissemination in mesh-based systems bears much randomness and uncertainty, both of them cannot guarantee the availability of streaming data by retrieving data from only local neighbors.

Distributed Hash Table (DHT) is a structure for managing

Manuscript received January 10, 2013; revised March 22, 2013.

Chen Zhuo is with National Key Laboratory of Science and Technology on Communications, University of Electronic Science and Technology of China. He is also a faculty member of College of Computer Science and Engineering, Chongqing University, China (e-mail: chenzhuo@ cqut.edu.cn).

Feng Gang is with National Key Laboratory of Science and Technology on Communications, University of Electronic Science and Technology of China, China (e-mail: fenggang@ uestc.edu.cn).

Lu Yi is with Huawei Technology, China (e-mail: uestccz@gmail.com). information and has been deployed in many file sharing applications. DHT can provide scalable and practical solutions to storing, locating and retrieving widely dispersed information in huge distributed network environments in certain number of hops. According to the different hop counts, DHTs can be classified into One-hop [1]-[3] and Multi-hop DHTs, which represent different trade-offs between lookup performance and maintenance overhead. Multi-hop DHTs aim at keeping a partial routing table to minimize the maintenance overhead, while the cost is the degradation of lookup performance after contacting several peers in sequence. On the contrary, One-hop DHTs maximize the lookup performance at the cost of maintaining a bigger (global) routing table.

To overcome the drawbacks of current P2P streaming systems, some previous work [4], [5] has taken DHT technology into consideration. However, most of the research work does not pose any limitation on which DHT systems to be used and often utilizes some common Multi-hop DHTs, such as Chord, Pastry, etc, which are of low search efficiency and/or high-latency. How to improve the searching efficiency of DHT for real-time streaming applications has not been carefully addressed. Especially considering the relevant traits of $\mathrm{P} 2 \mathrm{P}$ live streaming systems, there are still some challenges and problems in applying DHT in the following aspects:

1) Time-sensitivity: $P 2 P$ live streaming is a kind of real-time applications and the streaming data must be retrieved in time in order to guarantee the playback continuity. One-hop DHT instead of conventional multi-hop DHT is more appropriate in this sensitive scenario because of its high lookup efficiency.

2) Overlapping: The most important difference between P2P Live Streaming and VoD is that the playback position is relatively concentrated in the former. Peers always have some overlapping data in the buffer. Considering the use of DHT, it also means that the number of Indexes of a specific overlapping data will be large in the network. It is very inefficient to store and maintain redundant Indexes of a same data.

3) Availability: To avoid single point failure, replication mechanisms are often used in DHT to improve the availability of Indexes. For example, in Chord and D1HT, a peer will replicate all the Indexes to the following $\log _{2}{ }^{\mathrm{N}}$ successors, where $N$ is the network size. Although these traditional mechanisms are efficient, they will heavily burden the overhead in replication and storage.

In this paper, we propose a new highly efficient One-hop DHT lookup framework called Streaming-DHT (SDHT), aiming at improving the performance of fast sharing and searching for P2P live streaming applications. Considering 
the constraints and problems in the existing DHTs, in SDHT, we propose a modified solution called $\mathrm{EDRA}^{+}$to implement the One-hop DHT overlay, based on D1HT and EDRA. Besides, a Distributed Index Mapping and Management (DIMM) mechanism is designed to decrease the storage overhead and enhance the availability of Indexes without extra replication overhead. We selectively store the Indexes with better resources to decrease the storage overhead without performance degradation. To the best of our knowledge, this is the first work that leverages One-hop DHT and distributed Index mapping to optimize the use of DHT technology in $\mathrm{P} 2 \mathrm{P}$ live streaming systems.

The rest of this paper is organized as follows. Section II briefly introduces some related work. Section III proposes a new efficient One-hop DHT lookup framework called SDHT for P2P live streaming system, with emphasis on overlay implementation and optimization for Index mapping and storage. We present simulation results to evaluate the performance of our proposed SDHT in Section IV and finally conclude our paper in Section V.

\section{RELATED WORK}

DHT is a scalable, efficient, robust and self-organizing overlay routing infrastructure. DHTs have been successfully deployed in many large-scale peer-to-peer applications, such as distributed file sharing, communication, etc. Recently, they are also used to enable P2P streaming applications, such as live streaming and on-demand video streaming.

Haiying Shen et al. [4] propose a DHT-aided Chunk-driven Overlay (DCO). In DCO, they use a hierarchical DHT-based infrastructure and classify the peers into stable peers and common peers, where the stable peers are organized to form the DHT and other peers just choose one of stable peers to connect. DCO utilizes Chord as its DHT algorithm and the search latency is $O(\log N)$. In a related work called VMesh, K Yiu et al. [5] use a location aware DHT for searching suitable data providers. VMesh does not specify the DHT algorithm. In both DCO and VMesh, all the data segments stored in peers' buffer need to be registered on the DHT overlay. Therefore, large overhead will be incurred for registering and storing all the segments index information.

For the fast DHT lookup with one hop, some classic One-hop DHTs have been proposed such as D1HT [1], 1h-Calot [2] and OneHop [3]. A comprehensive comparison between these One-hop DHTs can be found in [7], which shows that D1HT consistently has the smallest overhead and better performance in load balancing than 1h-Calot and OneHop. D1HT uses the Event Detection and Reporting Algorithm (EDRA) algorithm to propagate the peer join/leave event. The design goal of D1HT is to optimize the searching performance with reasonable maintenance traffic overhead even for huge and dynamic P2P systems.

\section{STREAMING-DHT (SDHT) FRAMEWORK}

\section{A. EDRA+Design}

In existing single hop DHT frameworks, D1HT proposal has shown its competitive advantages in both load balancing and maintenance overhead. Although our proposed SDHT has some similarity with D1HT in overlay implementation and the theoretical aspect of D1HT is very clear, some implementation issues have to be addressed, which can stale routing table entries and lead peers to miss events. More importantly, errors in a peer's routing table can lead to the propagation of more errors in other peers' routing tables, which may substantially degrade the DH1T lookup performance. So the D1HT implementation issues should be carefully addressed in order to reduce stale routing table entries. In this paper, compared with D1HT, EDRA, EDRA* [6], [7] and D1HT*[8], we propose a modified solution called $\mathrm{EDRA}^{+}$to fulfill the One Hop DHT implementation.

We elaborate our solution EDRA+. EDRA+ is dedicated to addressing three major issues: 1 . How to join the network, which is simple but very important to the correctness of event propagation. 2. How to guarantee the correctness of event propagation. 3. How to handle the ACK related errors. Although some of these issues are also involved in other schemes such as [6]-[8], they are not complete.

\section{1) Join Mechanism}

The original D1HT does not detail the joining mechanism, leaving it to be specified at the implementation level, while D1HT* and EDRA* do not take it into consideration either. Work [7] uses a variant of Chord joining protocol and the only difference is that the new peer $P$ gets the full routing table from its successor $P_{\text {successor }}$. We also use a Chord like joining protocol with some differences. Unlike the other solutions, we use a HINT_MESSAGE to notify the old predecessor (relative to $P_{\text {successor }}$ ) about the new peer's coming when $P_{\text {successor }}$ accepts $P$ 's inserting. Because of this, $P_{\text {successor }}$ and the old predecessor should be the first peers to know about $P$ ' $s$ joining, which is an important factor for $\mathrm{EDRA}^{+}$to solve the events missing problem during propagation. Receiving the HINT_MESSAGE, the old predecessor does not only update its successor but also provides a copy of routing table to feed $P$ 's. Finally, the old predecessor becomes the new peer $P$ 's predecessor.

In EDRA* and work [7], because there is no priority for $P$ ' $s$ predecessor $E$ to know about the joining operation in advance, $P$ will observe it in at most $\Theta^{*} \rho$ time. These aforementioned three errors are all likely to occur in this situation. The solutions used in EDRA* and [7] are to forward all the events received by $P_{\text {successor }}$ or $P_{r t}$ to the new peer in a period of time after joining, where $P_{r t}$ is the peer who feeds $P$ 's routing table in EDRA*. According to the consequences of these errors, the solutions are only effective in the first kind of error and do not work well for the two other situations. In addition, duplicate events will increase with a high probability in these solutions.

D1HT* makes an assumption that $P$ 's successor and predecessor should be the first peers to know about $P$ 's joining, although it has not detailed how to realize this in the join process. In our solution $\mathrm{EDRA}^{+}$, we use join mechanism and HINT_MESSAGE to achieve this goal in practice. The solution of D1HT* is to add the address information of peer $\operatorname{succ}\left(p_{\text {any }}, 2^{l+1}\right)$ to each message with $T T L=l$ sent from peer $p_{\text {any }}$, $0 \leq l<\rho$. The receiving peer will realize that these events in the message should be forwarded to range $\left[\operatorname{succ}\left(p_{a n y}, 2^{l}\right)\right.$, 
$\left.\operatorname{succ}\left(p_{\text {any }}, 2^{l+1}\right)\right)$ and then adjust the number and TTLs of messages to send. However, in the first period, peer $D$ does not know peer $P$ and then sends three messages to $E, F, B$ with solid arrows. Peer $F$ will receive the message with $T T L=1$ from $D$. Since peer $F$ knows the new peer $P$, it will realize that the event should be forwarded to peer $P, A$ and then recalculate the number and TTLs of messages. In the second period, peer $F$ will send out two messages with long dotted arrows in the figure. Peer $P$ will not miss the event but $A$ will forward the event to $B$ in the third period. In the consequence, duplicate events will appear in peer $B$.

To cope with this problem, we add some address boundary information to each event instead of message. Assume that the peer who is propagating the events is $p_{a n y}$. When an event is new created but not being forwarded, boundary information in this event is the address of peer $\operatorname{succ}\left(p_{\text {any }}, 2^{l+1}\right)$, where $l$ is the TTL of certain message including the event. Peer records each event and the add-on boundary information in messages received in the last period. According to the recorded address boundary information of each event, peer adjusts the number and TTLs of messages to send. When an event in the message is being forwarded, the boundary information of this event is the address recorded last time and has nothing to do with the TTL of message any more.

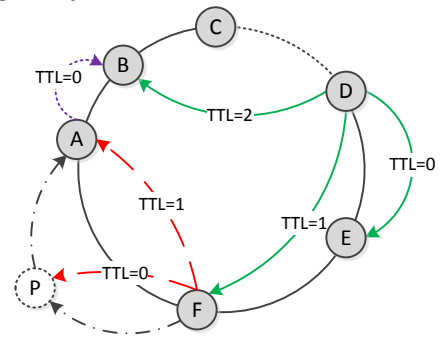

Fig. 1. Problem occurs in D1HT* solution

\section{2) ACK Mechanism}

D1HT* and work [7] have not given any solutions if the messages are unacknowledged. In our $\mathrm{EDRA}^{+}$, because the message may be lost due to link error or network congestion, we retransmit the unacknowledged messages for some times first. If the message is still unacknowledged after this, EDRA ${ }^{+}$ considers that the destination peer has failed and forwards message to the next peer in the routing table.

Another typical problem about the ACK mechanism occurs when a peer fails after receiving and acknowledging a message from another peer but this failure happens before this peer has forwarded the events received. The peer who sends the message will not try to retransmit it again after it has received the acknowledgement of this message. The propagation chain is broken and some other peers in the subsequent hops will miss the events included in this loss message. Similar to that in D1HT*, we also use Double-ACK mechanism in $\mathrm{EDRA}^{+}$to solve this problem. Any message sent with $T T L>0$ requires two acknowledgements to guarantee its correct forwarding.

\section{B. Distributed Index Solution}

Description of Index: When streaming server generates a new media unit or a peer receives a new media unit from other peers, an Index for this specific media unit will be created. Then the Index will be forwarded to and recorded in a peer namely responsor.

$$
\begin{array}{|l|l|}
\hline \multicolumn{2}{|c}{\text { TABLE I: THE INDEX FORMAT OF SDHT }} \\
\hline \text { Key } & \text { Value(IP,Label,Lifetime,SpareBandwidth) } \\
\hline
\end{array}
$$

Table I shows the format of the Index in SDHT, which consists of two parts: Key and Value. Key is a mapping value of a specific media unit by performing SHA-1. Value consists of four items: IP (IP address of the peer who is buffering the media unit), Label (The exclusive identifier of a specific media unit), Lifetime (The estimated lifetime of this buffered media unit), and SpareBandwidth (The spare uplink bandwidth of the peer who creates this Index).

Distributed Index Mapping and Management: Index mapping and management play an important role in DHT. In conventional DHT, centralized mechanisms are usually used, and same data entries have the same Key and responsosr peer. If a responsor peer crashes down or ungraceful leaves the network, the Indexes which is manage by this peer become unavailable, leading to search failure for these Indexes pairs. Some DHT algorithms adopt replication method to keep multiple copies on different peers to increase the data availability [1], however the need of extra storage space and extra replicating overhead compromise the advantage.

In order to overcome the pitfalls of conventional method, we propose a novel Distributed Index Mapping and Management (DIMM) Mechanism for SDHT to increase the chunk availability without incurring additional replication overhead. First, we introduce a new value $I D$. Both $I D$ and Key are in the same identifier space and we generally call this identifier space as key space in DHTs. For the sake of effective distinguish, we use $I D$ to identify nodes and use Key to identify data entries. Second, we introduce Identifier Space Division to divide the whole Identifier (key) Space into i Sub-spaces logically. Let us use an example to illustrate Identifier Space Division and DIMM. We assume that the entire Identifier Space is $[0 \ldots 180)$ and $i=4$ which means the Identifier Space is partitioned into four Sub-spaces equally. Peer $N i$ belongs to one of the Sub-spaces according to it $I D$, e.g. N150 belongs to Sub-space4. If the whole Identifier (key) space is $\left[0 \ldots 2^{m}\right)$ and $i$ is the number of Sub-spaces, the Sub-space identifier $S I D$ is given by

$$
\begin{gathered}
S I D=\left\{k \mid I D \in\left[\left\lfloor\frac{2^{m}-1}{i}\right\rfloor \cdot(k-1), \ldots,\left\lfloor\frac{2^{m}-1}{i}\right\rfloor \cdot k\right)\right\}, 1 \leq k \leq i \\
I D=\operatorname{Hash}(I P)
\end{gathered}
$$

When a peer retrieves an integrated chunk with Label value $L_{N}$ and is ready to create an Index, it can calculate the Key of this chunk using Key=Hash $\left(L_{N} \times S I D\right)$.

Unlike the file sharing scenario, chunk instead of file is the sharing unit for P2P live streaming. Index often represents the existence of such sharing unit in peer's buffer. So the number of Indexes in live streaming is much larger than file sharing scenario. Considering the huge number of overall Indexes, we don't have to store and maintain all the Indexes which are pointing to the same chunk. We add some limitations on the responsor to store and exchange Indexes. The details are described in algorithm 1. 


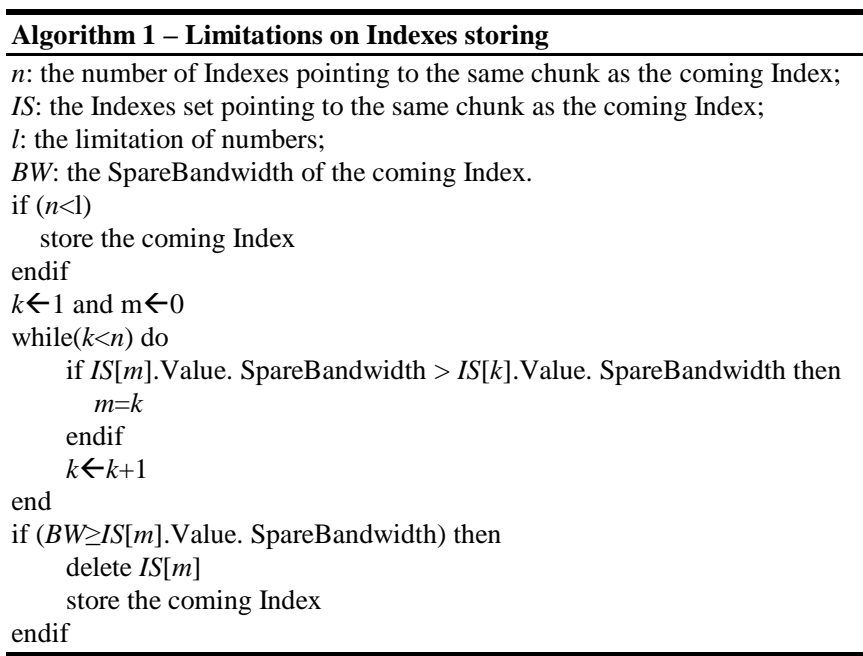

\section{Provider Locating in SDHT}

When a peer $p$ wants to search for a chunk with the Label value of $L_{N}$, it will first calculate the number of responsor peers it will send request to. As the number of Sub-spaces is $i$, there are at most $i$ responsor peers $p$ will send the request to at the same time. Intuitively sending search request simultaneously to multiple responsor peers can increase the searching success ratio and accelerate the searching process. The detailed searching algorithm using in SDHT is presented below.

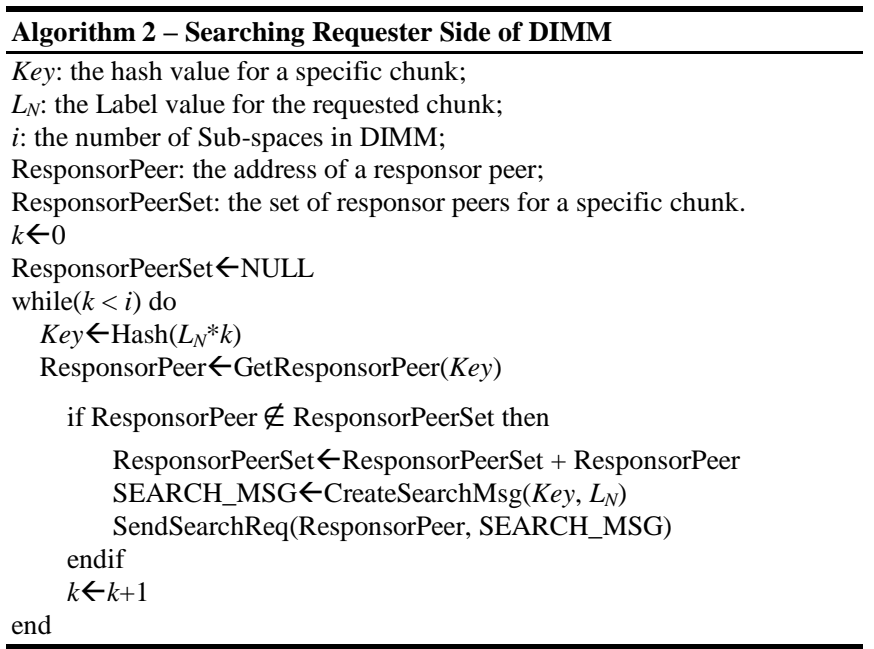

When a responsor peer in SDHT receives a specific SEARCH_MSG message, it will check its IndexTable for the specific SEARCH_MSG. Any Index matching the Label and Key in the message will becomes a candidate provider for the specific chunk, and the provider's relevant information will be replied from responsor peer to requester. The Responser Side Algorithm is described as follows.

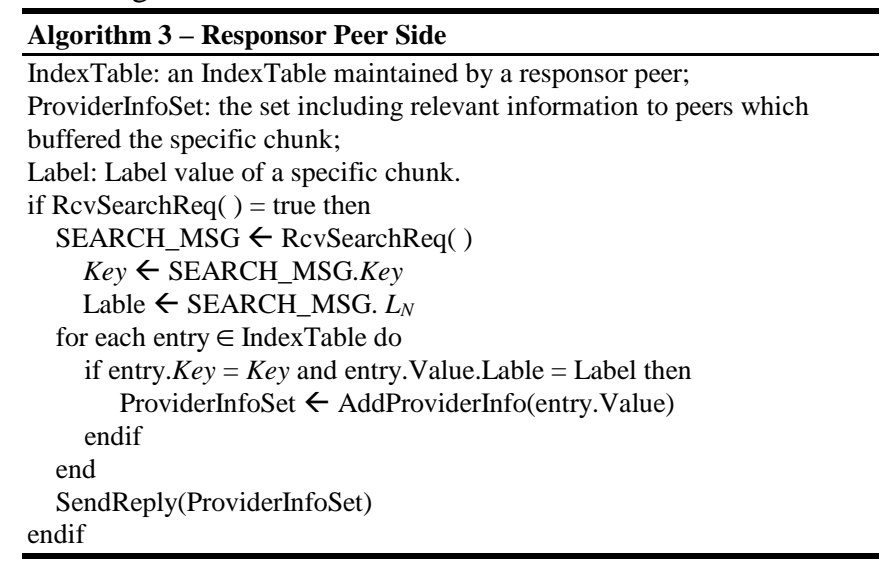

\section{Performance Evaluation And Discussions}

\section{A. Simulation Methodology}

To evaluate the performance of SDHT, we apply it to Denacast[9], a mesh-based P2P live streaming system implementation based on OverSim[10], and realize a live streaming system In our simulations, the average lifespans of peers are respectively 600 seconds (for CPs) and 3600 seconds (for SPs). We assess the impact of the optimizations in the proposed SDHT in this paper on search efficiency in P2P live streaming applications.

\section{B. Numerical Results}

Fig. 2 compares the search efficiency of Chord and our proposed SDHT, in terms of average hops needed in each search operation. In this experiment, the network size is set from 200 to 3200 . It is clear to show that almost all of the search requests are routed in one hop by using SDHT. The hop counts only slightly increase when network size changes from 200 to 3200. On the other hand, the average number of hops in Chord solution increases very fast with the network size. When the number of nodes is 3200 , the average hop counts in Chord are up to 6.92, which are much larger than in that in SDHT. Thus SDHT significantly outperforms Chord in search efficiency.

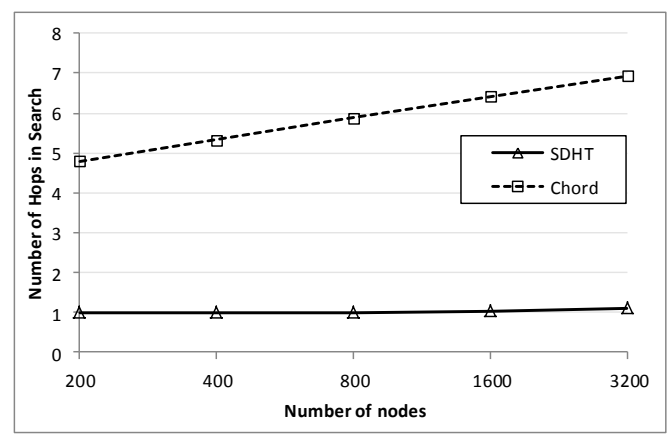

Fig. 2. Average number of hops in search

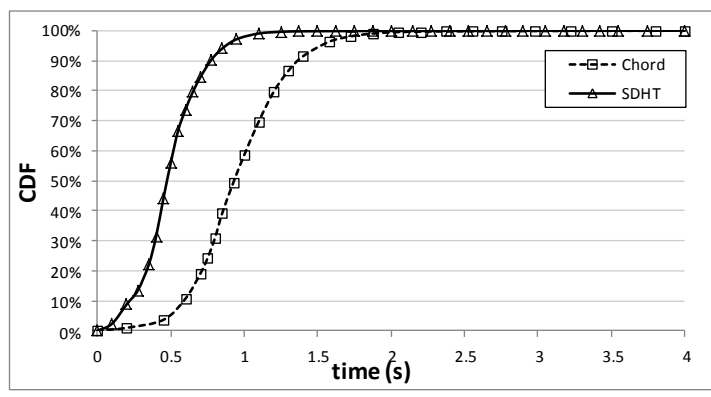

Fig. 3. CDF of compensation delay between Chord and SDHT

In Fig. 3, we evaluate the search efficiency of Chord and SDHT with another performance metric: Compensation Delay, which is defined as the search delay plus retrieving delay. Search delay is the time used for locating appropriate data provider and retrieve delay is the time interval from sending data request to receiving required data. Both Chord and SDHT are used to compensate the missing data in our simulation. The network size is 2200 , where the ratio of the number of SPs to that of CPs is $1 / 10$. The results show that the average compensation delay of Chord is almost twice than that of SDHT. We also see that $99 \%$ compensation delay in Chord solution is within $2.0 \mathrm{~s}$, while it is larger than $1.0 \mathrm{~s}$ in SDHT. The low compensation delay performance is very useful for time-sensitive applications like our $\mathrm{P} 2 \mathrm{P}$ live 
streaming system.

In the next experiment, we examine the impact of the number of sub-spaces or the maximum number of responsors on search efficiency in DIMM. Fig. 4 shows the comparison of the number of search failures in 280 s of simulation time. In this experiment, the data collection begins at 40s after the network becomes stable. When $i=1$, it means no space partitioning and the conventional Index mapping is employed. We can observe that DIMM has significantly smaller number of search failures than conventional Index mapping mechanism for all $i$ 's. The number of search failures decreases with $i$. This is because with the increase of the number of responsor peers, the impact of single node failure on search success probability decreases.

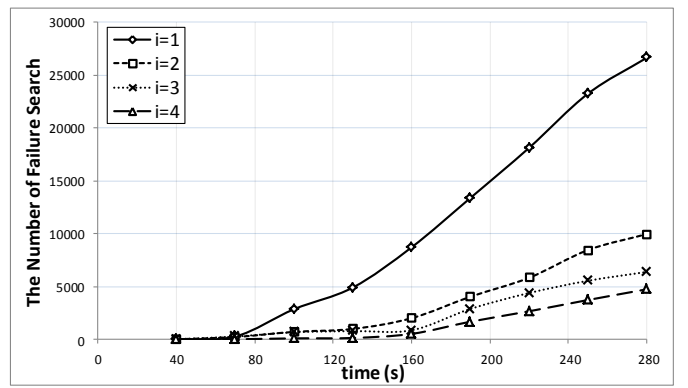

Fig. 4. Failure Search count with different number of responsors

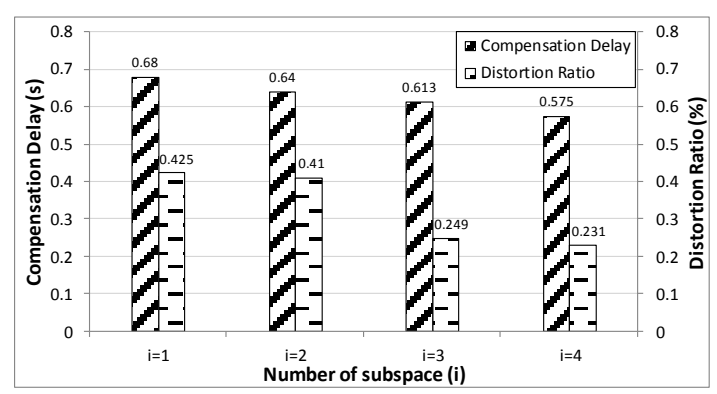

Fig. 5. Compensation Delay and Distortion Ratio for different number of responsors

Fig. 5 describes the compensation delay and distortion ratio as a function of the number of responsors, where distortion ratio is defined as the percentage of loss data which cannot be used by the player. We can see that when $i$ become bigger, the compensation delay decreases. It is because of that the number of simultaneous search requests becomes larger accordingly and simultaneous requests can accelerate the searching process. On the other hand, higher overhead will be incurred and therefore $i$ should not be too large. Without using the SDHT based compensation, the distortion ratio is up to $3.5 \%$. It falls below $0.45 \%$ while using the compensation mechanism. The distortion also decreases with $i$ due to the increase of successful search ratio. These results illustrate that the SDHT based compensation mechanism in HQStreaming can effectively improve playback quality and continuity.

\section{CONCLUSIONS}

In this paper, we have proposed an efficient One-hop DHT lookup framework for P2P live streaming systems which can offer higher search efficiency and data availability. For the time-sensitive applications, SDHT realizes a single hop DHT through $\mathrm{EDRA}^{+}$. In SDHT, we have designed DIMM to guarantee the high availability of streaming data and corresponding Indexes. DIMM can help save the storage space and further accelerate search process without incurring extra storage and replication overhead. These features make SDHT suitable for media data compensation for real-time streaming applications.

\section{ACKNOWLEDGEMENTS}

This work was supported by Key Projects for Science and Technology Development (No. 2009ZX03004-002) and National Science and Technology Fundamental Project (No. 2008ZX03003-005). Science \& Technology Research Project of Chongqing Education Committee (KJ120825)

\section{REFERENCES}

[1] L. Monnerat and C. Amorim, "D1HT: A distributed one hop hash table," in Proc. of IPDPS, Apr 2006.

[2] C. Tang, M. J. Buco, R. Chang, S. Dwarkadas, L. Luan, E. So, and C. Ward, "Low traffic overlay networks with large routing tables," in Proc. of SIGMETRICS, Jun 2005.

[3] P. Fonseca, R. Rodrigues, A. Gupta, and B. Liskov, "Full information lookups for peer-to-peer overlays," IEEE Transactions on Parallel and Distributed Systems, vol. 20, no. 9, Sep 2009.

[4] H. Shen, L. Zhao, Z. Li, and J. Li, "A DHT-aided chunk-driven overlay for scalable and efficient peer-to-peer live streaming," 2010 39th International Conference on Parallel Processing (ICPP), pp. 248-257, 13-16 Sept. 2010

[5] W.-P. K. Yiu, X. Jin, and S.-H. G. Chan, "VMesh: Distributed segment storage for peer-to-peer interactive video streaming," IEEE Journal on Selected Areas in Communications, vol. 25, no. 9, pp. 1717-1731, December 2007

[6] J. Buford, A. Brown, and M. Kolberg, "Analysis of an active maintenance algorithm for an O(1)-hop overlay," in Proc. GLOBECOM '07. IEEE Global Telecommunications Conference, pp. 81-86, 26-30 Nov. 2007

[7] L. Monnerat and C. Amorim, "Peer-to-peer single hop distributed hash tables," in Proc. IEEE Global Telecommunications Conference, pp. 1-8, Nov. 30 2009-Dec. 42009

[8] L. Monnerat and C. Amorim, "D1HT: A distributed one hop hash table (extended version)," COPPE/UFRJ, No. ES-705/06. 2006

[9] Denacast. [Online]. Available: http://denacast.org/

[10] I. Baumgart, B. Heep, and S. Krause, "OverSim: A flexible overlay network simulation framework," in Proc. of 10th IEEE Global Internet Symposium (GI '07) in conjunction with IEEE INFOCOM 2007, pp. 79-84, May 2007.

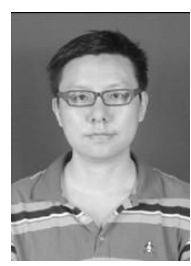

Chen Zhuo received the B.S. and M.S. degrees in Computer Science and Engineering from Chongqing University in 2002 and 2006, respectively. At present, he is a Ph.D. candidate at the National key Laboratory of Science and Technology on Communications, University of Electronic Science and Technology of China. His current research interests include $\mathrm{P} 2 \mathrm{P}$ networks and multimedia communications.

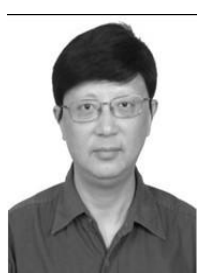

Gang Feng is a professor with the National Laboratory of Communications, University of Electronic Science and Technology of China. His research interests include resource management in wireless networks, wireless network coding, P2P networks, etc. Dr. Feng is a member of IEICE and senior member of IEEE.

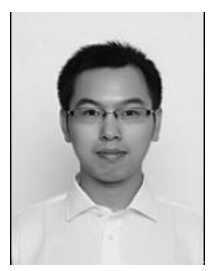

Lu Yi received the B.S. and M.S. degrees in Electrical Engineering from the University of Electronic Science and Technology of China (UESTC), Chengdu, in 2009 and 2012, respectively. His research interests include $\mathrm{P} 2 \mathrm{P}$ network and multimedia communications. 